\title{
Cirugía Laparoscópica en el Cáncer de Colon, en el Hospital Oncológico Solca- Quito.
}

*Correspondencia:

drrueda72@hotmail.com

Teléfono [593] 022419775

Conflicto de intereses: Los autores declaran no tener conflictos de intereses.

Fondos: Ver la página 109

Recibido: 26 Febrero 2017

Aceptado: 20 Julio 2018

Publicado: 30 Agosto 2018

Membrete bibliográfico: Rueda M, Peralta P, Mesías C. Cirugía Laparoscópica En el Cáncer de Colon, en el Hospital Oncológico Solca-Quito. Rev. Oncol. Ecu 2018;28(2):103-111.

DOI: https://doi.org/10.33821/125

Copyright Rueda, et al. Este artículo es distribuido bajo los términos de Creative Commons Attribution License, el cual permite el uso y redistribución citando la fuente y al autor original.

\section{Laparoscopic Surgery in Colon Cancer, at the Solca-Quito Oncological Hospital.}

\author{
Miguel Rueda Mesías1* (D), Patricio Peralta1, Carolina Mesías1.
}

1. Hospital Oncológico "Solón Espinosa Ayala". Solca Núcleo de Quito, QuitoEcuador, Servicio de Cirugía Oncológica.

\section{Resumen}

Introducción: La cirugía para el cáncer de colorrectal consiste en la resección en bloque del tumor y de $\geq 12$ ganglios linfáticos regionales, debe incluir la ligadura alta del vaso principal del segmento. Este conjunto de técnicas pueden ser realizadas por vía laparoscópica y el objetivo del presente estudio es presentar los resultados de un centro único con el abordaje quirúrgico laparoscópico en un grupo de pacientes diagnosticados con cáncer de colorrectal.

Métodos: En el departamento de Cirugía Oncológica del Hospital "Solón Espinosa Ayala" de Quito, durante el período Enero del 2009 a Septiembre 2012 se realizó un estudio descriptivo, retrospectivo. Se analizaron todos los casos de pacientes derivados del área con diagnóstico inicial de tumor de colorectal, a los cuales previo a realizarles marcadores tumorales, Tomografía de Tórax-abdomen, y a quienes se les realizó como método diagnóstico colonoscópico. Se excluyeron pacientes con neoplasias de origen secundario, con historias clínicas incompletas que imposibilitaron el análisis. Se estudiaron las variables sexo, edad, localización del tumor, diagnostico histopatológico y morbilidad perioperatoria. El análisis estadístico realizado fue descriptivo.

Resultados: Se evaluaron 25 pacientes con diagnóstico tumor de mediastino, con una edad media de 61 años. Fueron 15 mujeres (60 \%). El estudio histopatológico post-cirugía fue 10 casos (40\%) con adenocarcinoma moderadamente diferenciado, 7 casos (28 \%) con adenocarcinoma bien diferenciado. La resección de $\geq 12$ ganglios linfáticos regionales se realizó en 15 casos ( $75 \%$ ). En 13 casos (52\%) recibieron Adyuvancia. 1 caso requirió conversión a cirugía abierta. Morbilidad se presentó en 2 casos (8 \%) con fístula y 1 caso (4 \%) neumonía.

Conclusión: El adenocarcinoma fue el tumor maligno más frecuente en esta serie. El manejo quirúrgico laparoscópico de tumores colorectales tuvo una baja tasa de complicaciones y un abordaje quirúrgico en el 75 \% de los casos con resección de más de 12 ganglios peritumorales.

Palabras Claves: NEOPLASIAS DE COLON, NEOPLASIAS COLORRECTALES, LAPAROSCOPÍA.

DOI: $10.33821 / 125$ 


\section{Abstract}

Introduction: Surgery for colorectal cancer consists of resection in the tumor block and $\geq 12$ regional lymph nodes, it must include the ligation of the main vessel of the segment. This set of techniques can be laparoscopically performed and the objective of this study is to present the results of a single center with the surgical laparoscopic surgical approach in a group of patients diagnosed with colorectal cancer.

Methods: In the Department of Oncological Surgery of the Hospital "Solón Espinosa Ayala" of Quito, a descriptive, retrospective study was carried out during the period from January 2009 to September 2012. All cases of patients with the initial diagnosis of colorectal tumor, previous examinations, tumor markers, chest-abdomen tomography, and those that have been developed as a colonoscopic diagnostic method will be analyzed. We excluded patients with neoplasms of secondary origin, with incomplete clinical histories that make analysis impossible. The variables sex, age, tumor location, histopathological diagnosis and perioperative morbidity will be studied. The statistical analysis performed was descriptive).

Results: 25 patients with mediastinal tumor diagnosis were evaluated, with an average age of 61 years. There were 15 women (60\%). The postoperative histopathological study had 10 cases (40\%) with moderately differentiated adenocarcinoma, 7 cases (28\%) with well differentiated adenocarcinoma. Resection of $\geq 12$ regional lymph nodes was performed in 15 cases (75 \%). In 13 cases (52\%) they received adjuvant. 1 case required conversion to open surgery. Morbidity occurred in 2 cases ( $8 \%$ ) with fistula and 1 case (4\%) pneumonia.

Conclusion: Adenocarcinoma was the most frequent malignant tumor in this series. Laparoscopic surgical management of colored tumors had a low complication rate and a surgical approach in $75 \%$ of the cases with resection of more than 12 peritumoral lymph nodes.

Keywords: COLON NEOPLASIAS, COLORECTAL NEOPLASMS, LAPAROSCOPY.

DOI: $10.33821 / 125$

\section{Introducción}

El tratamiento quirúrgico del cáncer de colon y recto ha tenido importantes avances en la última década. La resección quirúrgica del tumor primario con márgenes de resección adecuados y la linfadenectomía correspondiente proporciona una mejor oportunidad de supervivencia libre de enfermedad a largo plazo [1]. La colectomía abierta convencional es considerada como el tratamiento estándar para las enfermedades benignas y malignas [2]. La colectomía laparoscópica se describió en los años 90 del siglo $X X$ y, aunque es técnicamente desafiante, constituye una opción factible para la resección del colon. A medida que las técnicas y las tecnologías avanzan, la cirugía laparoscópica ha sido mejor aceptada y es más comúnmente usada en muchas instituciones debido a las ventajas descritas: reducción del dolor postoperatorio, menor tiempo de recuperación, mejor resultado cosmético y menor tiempo de hospitalización [3-4].

La cirugía para el cáncer de colon consiste en la resección en bloque del tumor y de los ganglios linfáticos regionales, debe incluir la ligadura alta del vaso principal del segmento; es decir de la base del meso a la raíz del meso arterial para poder extirpar todos los ganglios 
del mesenterio; y la resección mínima de 12 ganglios linfáticos en la pieza, para considerarse como un procedimiento adecuado, ya que, hoy por hoy, los ganglios siguen siendo el factor pronóstico más importante para la decisión de tratamiento adyuvante, y para ofrecer una sobrevida mayor, y un tiempo mayor libre de enfermedad [5-7]. Este conjunto de técnicas pueden ser realizadas por vía laparoscópica y el objetivo del presente estudio es presentar los resultados de un centro único con el abordaje quirúrgico laparoscópico en un grupo de pacientes diagnosticados con cáncer de colon.

\section{Materiales y Métodos}

En el departamento de Jefatura de Cirugía Oncológica del Hospital "Solón Espinosa Ayala" Solca-Quito, durante los meses de Enero del 2009 a Septiembre de 2012 se realizó un estudio descriptivo, retrospectivo. Se analizaron todos los casos de pacientes derivados del área de pre admisión con diagnóstico inicial de Cáncer de colorrectal, a los cuales previo a realizarles marcadores tumorales, Tomografía de Tórax-abdomen para estadiaje, a quienes se les realizó como método diagnóstico colonoscopía y en como método terapéutico abordaje quirúrgico laparoscópico.

Los criterios de inclusión fueron pacientes de ambos sexos mayores de 18 años, con diagnóstico de tumor colorrectal y que recibieron tratamiento quirúrgico en el departamento de Jefatura de Cirugía Oncológica de Solca-Quito. Se excluyeron pacientes con neoplasias de origen secundario, con historias clínicas incompletas que imposibilitaron el análisis.

Se estudiaron las variables sexo, edad, Tipo de Técnica quirúrgica, localización del tumor, diagnostico histopatológico y mortalidad perioperatoria. El análisis estadístico realizado fue descriptivo con el paquete estadístico Epi Info-2008.

\section{Resultados}

Se evaluaron 25 pacientes en el período de estudio. De total de casos analizados, 15 casos (60\%) fueron mujeres y 10 casos ( $40 \%$ ) fueron hombres. El promedio de edad fue 61 años, con rangos de edad desde 21 años hasta 84 años. 8 casos (32\%) con antecedentes médicos de Hipertensión arterial esencial, Diabetes tipo2, Arritmias y Obesidad.

Los diagnósticos prequirúrgicos, se obtuvieron mediante la toma de muestra en la colonoscopia. Los más prevalentes fueron el adenocarcinoma bien diferenciado y moderadamente diferenciado (Tabla 1). Previo a la cirugía también se consideró la descripción de TAC en cuanto a la presencia o no de ganglios. Solamente en dos casos (8 \%) se reportó ganglios presentes, adyacentes al tumor. Posterior a la cirugía 8 casos (32 \%) se reportaron ganglios positivos para metástasis en el estudio histopatológico. De los 25 casos, solamente uno (4\%) recibió terapia neoadyuvante (Quimioterapia-Radioterapia). En cuanto al tipo de cirugía la Hemicolectomía izquierda fue el tipo de cirugía más prevalente, 
seguida de la sigmoidectomía (Tabla 2). Podemos ver que según el resultado del histopatológico definitivo, de los 25 casos sometidos a cirugía, 20 tuvieron un diagnóstico de malignidad y 5 casos fueron lesiones benignas (Tabla 1). De los 25 casos 15 ( $75 \%$ fueron reportadas en patología como mayores a 12 ganglios incluidas en la muestra de patología. El promedio del tiempo quirúrgico en las cirugías fue de 150 minutos.

Tabla 1. Diagnósticos histopatológicos Prequirúrgicos y postquirúrgicos

\begin{tabular}{|l|c|c|}
\hline Diagnóstico Histopatológico & $\begin{array}{c}\text { Diagnóstico } \\
\text { Pre- } \\
\text { Quirúrgico } \\
\mathrm{n} 25(100 \%)\end{array}$ & $\begin{array}{c}\text { Diagnóstico } \\
\text { Post- } \\
\text { Quirúrgico } \\
\mathrm{n}=25(100 \%)\end{array}$ \\
\hline Adenocarcinoma moderadamente diferenciado & $6(24 \%)$ & $10(40 \%)$ \\
\hline Adenocarcinoma bien diferenciado & $8(32 \%)$ & $7(28 \%)$ \\
\hline Colitis / Inflamación crónica & - & $2(8 \%)$ \\
\hline Adenoma con displasia de Bajo Grado & $3(12 \%)$ & $1(4 \%)$ \\
\hline Adenocarcinoma In Situ & $1(4 \%)$ & $1(4 \%)$ \\
\hline Diverticulosis & $1(4 \%)$ & $1(4 \%)$ \\
\hline Adenocarcinoma residual en Muñón & - & $1(4 \%)$ \\
\hline Ausencia de tumor Residual & - & $1(4 \%)$ \\
\hline Lipoma Submucoso Colónico & - & $1(4 \%)$ \\
\hline Adenoma con Displasia de Alto Grado & $2(8 \%)$ & - \\
\hline Adenocarcinoma de apéndice & $1(4 \%)$ & - \\
\hline Ca de Cervix / Proctitis Actínica & $1(4 \%)$ & - \\
\hline Posible Gist & $1(4 \%)$ & - \\
\hline Tumor de Apéndice Carcinoide & $1(4 \%)$ & - \\
\hline
\end{tabular}

Tabla 2. Tipo de cirugía laparoscópica realizada

\begin{tabular}{|l|c|c|}
\hline Tipo de Cirugía Laparoscópica & $\begin{array}{c}\text { Frecuencia } \\
\mathbf{n = 2 5}\end{array}$ & $\begin{array}{l}\text { Porcentaje } \\
\mathbf{1 0 0 \%}\end{array}$ \\
\hline Hemicolectomía Izquierda & 8 & $32 \%$ \\
\hline Sigmoidectomía & 6 & $24 \%$ \\
\hline Hemicolectomía Derecha & 4 & $16 \%$ \\
\hline Resección Anterior Baja & 4 & $16 \%$ \\
\hline Resección Anterior Ultrabaja & 2 & $8 \%$ \\
\hline Colectomía total & 1 & $4 \%$ \\
\hline
\end{tabular}

Tabla 3. Adyuvancia post-quirúrgica

\begin{tabular}{|l|c|c|}
\hline Ninguna & $\begin{array}{c}\text { Frecuencia } \\
\mathbf{N}=25\end{array}$ & $\begin{array}{c}\text { Porcentaje } \\
100 \%\end{array}$ \\
\hline QT & 9 & $48 \%$ \\
\hline QT / RT & 2 & $36 \%$ \\
\hline RT & 2 & $8 \%$ \\
\hline
\end{tabular}


QT: Quimioterapia; RT: Radioterapia

Las complicaciones posquirúrgicas fueron en 3 casos, 2 casos fueron fistulas en las anastomosis, que se controlaron con cirugía y colostomía. Un caso presentó Neumonía. Un solo paciente presentó complicación durante el procedimiento quirúrgico, que al mismo tiempo fue la única cirugía con necesidad de conversión. En promedio los días de estancia hospitalaria fue de 5 días. Luego de la cirugía 13 casos (52\%) recibieron Adyuvancia postquirúrgica (Tabla 3).

\section{Discusión}

En la presente serie de casos, los pacientes con tumor colorrectal fueron la mayoría de sexo femenino, resultados que están en relación con los artículos publicados por la comunidad científica [8-12]. En cuanto a la edad, aunque se reporta que se presenta en cualquier etapa de la vida, en este reporte en los 4 años del registro, la edad media de estos pacientes es de 61 años.

Las estrategias terapéuticas quirúrgicas laparoscópicas de los tumores colorectales pueden ser la primera opción terapéutica, aunque su aplicación depende de la experiencia del cirujano y de su equipo; localización anatómica, etiología de la enfermedad, recursos institucionales y las comorbilidades acompañantes.

En este reporte de 25 casos el resultado patológico predominante en los tumores colorectales fue el Adenocarcinoma, lo que coincide con los reportes de la literatura internacional [13-18].

No se reportaron fallecidos en esta serie, por lo que es importante en las neoplasias colorectales un diagnóstico temprano que permita seleccionar la estrategia terapéutica más adecuada de los pacientes afectados, sobre todo con neoplasias malignas, en este reporte se incluyeron patologías benignas como colitis, diverticulitis y lipomas que en un primer acercamiento fueron consideradas como patología maligna por el estudio anatomopatológico tomada por colonoscopía. Futuras investigaciones deberán abordar seguimientos, supervivencias, técnicas con un grupo control para el mejor estudio del tema.

Un resultado quirúrgico con un abordaje bueno incluye la resección de más de 12 ganglios incluidos en la muestra de patología. El presente estudio demuestra que es posible en más de $75 \%$ de los casos intervenidos. La cirugía laparoscópica aparentemente tiene los mismo resultados a largo plazo que la cirugía convencional, y a corto plazo se han realizado múltiples investigaciones acerca de ciertos factores en los que hay franca diferencia de la cirugía abierta, como disminución de los días de hospitalización, movilidad y funcionamiento intestinal más temprano, reduce el dolor postoperatorio, y un mejoramiento de la calidad de vida a corto plazo, en este estudio no se presenta un grupo comparativo por lo que se plantea como idea de investigación para próximos estudios. 
La mayoría de los pacientes son candidatos para un tratamiento laparoscópico. Cuando el cirujano es experimentado, incluso los pacientes con una historia de cirugía abdominal previa se valoran como posibles candidatos. Según la revisión sistemática Cochrane [1], los beneficios a corto plazo de la cirugía laparoscópica en cáncer de colon y recto están ya descrito pero los beneficios a largo plazo como son la sobrevida, recurrencia y las complicaciones a largo plazo deben ser más estudiadas, ya que hasta el momento no han mostrado diferencias significativas.

Dentro de este estudio se analiza el número de ganglios resecados como factor importante, pero se observó que este factor no está relacionado solamente con el tipo de técnica quirúrgica usado, sino también con el tipo de técnica patológica que se usa para procesar la muestra. Sin embargo se establece que un número de ganglios menores a 12 nos lleva a un subestadiaje. Y si, se observa menor número de ganglios resecados por técnica laparoscópica que por técnica abierta. En el resto de complicaciones como incidencia de hernias, y adherencias postquirúrgicas aún faltan estudios.

Con todo esto podemos decir que hay suficiente evidencia sólida que indica que la resección laparoscópica de colon es una buena alternativa, con ventajas a corto tiempo, cuando es realizada por un cirujano experto con el paciente adecuado, puede alcanzar resultados aceptados para los estándares oncológicos, que incluye apropiada resección de márgenes, y número adecuado de ganglios. Pero a pesar de esto no hay datos que apoyen su superioridad frente a la técnica abierta.

\section{Conclusiones}

En conclusión el manejo quirúrgico de los tumores colorectales continúa siendo un reto para el paciente que lo padece y el equipo quirúrgico, debido a la diversidad de la patología y la complejidad del tratamiento. El adenocarcinoma fue el tumor maligno más frecuente en esta serie. Podemos finalmente establecer que de forma general, se obtuvieron resultados que concuerdan, en gran medida, con otros estudios realizados sobre manejo quirúrgico laparoscópico de tumores colorectales con una baja tasa de complicaciones y un abordaje quirúrgico en el $75 \%$ de los casos con resección de más de 12 ganglios peritumorales.

\section{Agradecimientos}

Se reconoce a las personas que participaron indirectamente en el estudio tales como el personal técnico y otras en general del Hospital "Solón Espinosa Ayala" de Solca-Núcleo de Quito. 


\section{Información adicional}

\section{Nota del Editor}

La Revista Oncología Ecu permanece neutral con respecto a los reclamos jurisdiccionales en mapas publicados y afiliaciones institucionales.
Abreviaturas

QT: Quimioterapia.

RT: Radioterapia.

Archivos Adicionales

Ninguno declarado por los autores.

Fondos

Los fondos de la investigación fueron propios de los autores del presente artículo.

\section{Disponibilidad de datos y materiales}

Existe la disponibilidad de datos bajo solicitud al autor de correspondencia. No se reportan otros materiales.

\section{Contribuciones de los autores}

MR, PP, realizaron la idea de investigación, revisión bibliográfica, el análisis crítico del artículo. CM realizó la recolección de datos, análisis estadístico, escritura del artículo. CM realizó las correcciones. Todos los autores leyeron y aprobaron la versión final del artículo.

Aprobación de ética y consentimiento para participar

No aplica ya que es un estudio retrospectivo

\section{Consentimiento para publicación}

No aplica.

\section{Información de los autores}

Miguel Rueda Mesías, Médico cirujano oncólogo, del Hospital "Solón Espinosa Ayala" Solca-Núcleo de Quito. (iD) https://orcid.org/0000-0001-6821-8418 
Patricio Peralta, Médico cirujano oncólogo, del Hospital "Solón Espinosa Ayala" SolcaNúcleo de Quito.

Carolina Mesías, cirujano general residente del servivio de Cirugía Oncológica, del Hospital "Solón Espinosa Ayala" Solca-Núcleo de Quito.

Revisiones por pares

Acceda a la revisión de pares académicos en el siguiente enlace: https://publons.com/review/3729470

\section{Referencias}

Abreviaturas en la referencias

DOI: Digital Object

Identifier

PMID: PubMed Identifier

SU: Short URL
1. Kuhry E, Schwenk W, Gaupset R, Romild U, Bonjer HJ. Long-term results of laparoscopic colorectal cancer resection. Cochrane Database of Systematic Reviews 2008;(2)CD003432. DOI: 10.1002/14651858.CD003432.pub2.

2. Labianca R, Nordlinger B, Beretta G.D, Brouquet A, Cervantes A. On behalf of the ESMO Guidelines Working Group; Primary colon cancer: ESMO Clinical Practice Guidelines for diagnosis, adjuvant treatment and follow-up, Annals of Oncology 2010; 21(5):v70-v77. DOI: 10.1093/annonc/mdq168

3. Waters J, Chihara R, Moreno J, Robb B, Wiebke E, George V. Journal of the Society of Laparoendoscopic Surgeons Laparoscopic Colectomy: Does the Learning Curve Extend Beyond Colorectal Surgery Fellowship?, JSLS. 2010;14(3): 325-331. DOI: 10.4293/108680810X12924466006800

4. Wasserberg N. Laparoscopic Colectomy for colorectal cancer. The Israel Medical Associatin Journal, 2010; 12(9):572-576.

5. Veldkamp R, Kuhry E, Hop WC, Jeekel J, Kazemier G, Bonjer HJ, et al. Survival after laparoscopic surgery versus open surgery for colon cance: long-term outcome of a randomized clinical trial. Lancer Oncol 2015; 6:477-484. DOI: 10.1016/S1470-2045(05)70221-7

6. Braga M, Frasson M, Vignali A, Zuliani W, Capretti G, Di Carlo V. Laparoscopic resection in rectal cancer patients: outcome and cost-benefit analysis Dis Colom rectum. 2007;50:464-471. DOI: $10.1007 / \mathrm{s} 10350-006-0798-5$

7. Guerrieri M, Campagnacci R, De Sanctis A, Lezoche G, Massucco P, Summa M, Gesulta R, et al Laparoscopic versus open colectomy for TNM stage III colon cancer: results of a prospective multicenter study in Italy. Surg Today November 2012;42:1071-1077. DOI: 10.1007/s00595-012-02928.

8. Ibáñez Aguirre F, Almendral López M, Clemares de Lama M. Cirugía del carcinoma de colon por vía laparoscópica. Oncología (Barc.). 2004;27(4):121-123. DOI: 10.4067/S0718-40262008000300004

9. Zárate A, López-Köstner F, Loureiro C, Pinedo G, Molina M, Kronberg U, et al. Resultados y eventos adversos de la sigmoidectomía por cáncer: laparoscopia versus laparotomía. Rev Chil Cir. 2008; 60(1): 29-34. DOI: 10.4067/S0718-40262008000100007.

10. Abellán $M$, Balagué $C$, Pallarés $J$, Carrasquer $A$, Hernández $P$, Martínez $M$, et al. Factores de morbimortalidad postoperatoria tras cirugía laparoscópica por cáncer de colon en pacientes octogenarios, Cirugía Española 2012;90: 589-594. DOI: 10.1016/j.ciresp.2012.04.003

11. Gunka J, Dostalik J, Martinek L, Gunkova P, Mazur M, Vavra P, Long-term results of laparoscopic versus open surgery for nonmetastatic colorectal cancer, Acta Chir Belg. 2012;112(2):139-147. DOI: $\underline{10.1089 / \text { lap.2007.0169 }}$ 
12. Cortés S, Csendes A, Yarmuch J. Resultados de la unidad de oncología del Departamento de Cirugía de un hospital universitario (2004-2010). Rev Chil Cir, 2011;63(5):534-537. DOI: 10.4067/S071840262011000500017.

13. Koopmann MC, Heise $\mathrm{CP}$, Laparoscopic and minimally invasice resection of malignant colorectal disease, Surg Clin North Am, 2008;88(5):1047-1072. DOI: 10.1016/j.suc.2008.05.009.

14. Meredith KL, Hoffe SE, Shibata D. The multidisciplinary management of rectal cancer. Surg Clin North Am. 2009;89(1):117-215. DOI: 10.1016/j.suc.2008.09.021.

15. Chua T, Chong C, Liauw W, Morris D. Approach to Rectal Cancer Surgery International Journal of Surgical Oncology 2012;2012:247107. DOI: 10.1155/2012/247107

16. Jeong SY, Park JW, Nam BH, Kim S, Kang SB, Lim SB, et al. Open versus laparoscopic surgery for mid or low rectal cancer after neoadjuvant chemoradiotherapy (COREAN trial): survival outcomes of an openlabel, non-inferiority, randomized controlled trial. Lancet Oncol 2014;15(7):767-774. DOI: $\underline{10.1016 / S 1470-2045(14) 70205-0}$

17. Martel G, Crawford A, Barkun JS, Boushey RP, Ramsay CR, et al. (2012) Expert Opinion on Laparoscopic Surgery for Colorectal Cancer Parallels Evidence from a Cumulative Meta-Analysis of Randomized Controlled Trials Lancet Oncol 2010;11(7):637-645. DOI: 10.1371/journal.pone.0035292

18. Martel G, Duhaime S, Barkun JS, Boushey RP, Ramsay CR, Fergusson DA. The quality of research synthesis in surgery: the case of laparoscopic surgery for colorectal cancer. Syst rev 2012;17:1-14. DOI: 10.1186/2046-4053-1-14. 